Relations industrielles

Industrial Relations

\title{
Management Decisions and Behavior, by Max D. Richards and Paul S. Greelaw, Georgetown, Ontario, Irwin-Dorsey Limited, 1972, 655 pp.
}

\section{Jean-Marie Rainville}

Volume 28, numéro 3, 1973

URI : https://id.erudit.org/iderudit/028436ar

DOI : https://doi.org/10.7202/028436ar

Aller au sommaire du numéro

Éditeur(s)

Département des relations industrielles de l'Université Laval

ISSN

0034-379X (imprimé)

1703-8138 (numérique)

Découvrir la revue

Citer ce compte rendu

Rainville, J.-M. (1973). Compte rendu de [Management Decisions and Behavior, by Max D. Richards and Paul S. Greelaw, Georgetown, Ontario, Irwin-Dorsey Limited, 1972, 655 pp.] Relations industrielles / Industrial Relations, 28(3), 674-675. https://doi.org/10.7202/028436ar

Tous droits réservés @ Département des relations industrielles de l'Université Laval, 1973
Ce document est protégé par la loi sur le droit d'auteur. L’utilisation des services d'Érudit (y compris la reproduction) est assujettie à sa politique d'utilisation que vous pouvez consulter en ligne.

https://apropos.erudit.org/fr/usagers/politique-dutilisation/ 
sidered, it is not an exorbitant price. If we attempt to suppress strikes, we will find ourselves drifting in the direction of a dictatorial regime. The best prescription for strikes is to see to it that labor - and other groups - are not forced to strike.

\section{Jean BOIVIN}

\section{Université Laval}

Management Decisions and Behavior, by Max D. Richards and Paul S. Greenlaw, Georgetown, Ontario, Irwin-Dorsey Limited, 1972, 655 pp.

Ce manuel revisé d'introduction à l'administration demeure fidèle à la version originale parue en 1966 quant au cadre conceptuel et au contenu. Cependant, pour chacun de ces deux points, les auteurs ont mieux précisé leur approche en complétant par la notion de système le point de vue selon lequel ils abordent l'administration, soit celui de la décision tel que Simon le proposait. Ils élargissent ainsi leur cadre théorique initial.

Cependant, l'effort de renouvellement du manuel ne se détache pas de l'Ecole classique en administration. A cet égard, la notion de système est appliquée à l'organisation en tant que telle. Aussi, l'administration est présentée comme un soussystème quasi spécifique d'un ensemble plus vaste, ou de l'environnement. Mais les auteurs ne prennent pas en considération ce dernier concept d'une manière systématique dans un manuel. Pour y parvenir, ils essaient dans un effort ultime de faire appel au travail de Lawrence et Lorsch (Organization and Environment) mais ils oublient que l'environnement n'est traité par ces auteurs que du point de vue de ceux qui ont à prendre des décisions, et ne constitue pas un objet d'analyse spécifique. Le concept d'environnement demeure général et abstrait dans le manuel.

En ce sens, lorsque les auteurs essaient d'énoncer une théorie de l'administration en termes de «situation》 ou de «management conditionnel», ils demeurent prisonniers de leur méthode d'approche. Ils ne s'éloignent pas pour la peine des modèles d'analyse énoncés par Burns et Stalker (The Management of Innovation) dans leur analyse com- parative d'entreprises industrielles, et de Fiedler (A Theory of Leadership Effectiveness) pour l'analyse du leadership. En outre, dans ce dernier cas, la valeur de l'approche est loin d'être démontrée comme G. Strauss (Organizational Behavior and Personnel Relations. Reprint No 346, Calif. Institute of Industrial Relations, 1970), l'a fait remarquer à juste titre.

D'une manière plus générale, on pourrait dire que les auteurs prennerit trop facilement certaines conclusions de recherche pour définitivement acquises à propos desquelles les chercheurs concernés s'interrogent eux-mêmes. C'est surtout le cas pour tout le contenu des chapitres concernant l'individu, le leadership et le groupe. La limite des théories présentées de même que leurs implications ne sont pas discutées. A ce propos, la question est la suivante : jusqu'à quel point peut-on parler de l'individu en tant qu'individu ou en tant que membre d'un groupe (informel ou autre) sans tenir compte de la relation d'autorité où il est placé ? Si on ne le fait pas, l'aspect structurel de l'intégration de l'individu à l'organisation n'est pas traité, et bien sûr, la notion de conflit est évitée ou ignorée dans l'analyse.

La même faiblesse se reproduit lorsqu'il s'agit d'examiner les processus organisationnels au chapitre 8 . Les auteurs donnent l'une à la suite de l'autre deux thèses explicatives de la structure administrative, l'une fondée sur la stratégie administrative, l'autre sur la technologie. Bien que ces thèses soient aux antipodes l'une de l'autre, les auteurs ne s'en souviennent guère. Par ailleurs, les recherches ayant démontré que la structure administrative n'est pas dépendante de la technologie, et dont certaines sont parues vers le milieu des années ' 60 ne sont pas mentionnées.

Lorsqu'il s'agit d'énoncer des principes classiques du management ou techniques administratives les auteurs sont irréprochables, et demeurent fidèles à l'Ecole classique. Par contre, c'est justement l'utilisation de cette même démarche intellectuelle pour l'analyse de l'organisation administrative du point de vue des sciences du comportement, qui constitue la faiblesse majeure du manuel.

Etant donné nos réserves, si l'enseignement universitaire conserve la formation 
intellectuelle des jeunes comme objectif, le manuel, tel quel, n'a pas une très grande valeur. Il ne peut être utilisé à cette fin qu'à la condition que le cadre conceptuel de fond et le contenu soient présentés aux étudiants d'une manière critique. Si cette condition est remplie, il peut être un bon manuel.

\section{Jean-Marie RAINVILLE}

Université de Montréal

Operations Research for Managerial Decisions, by Donald R. Plane and Gary A. Kochenberger, Homewood, Ill., Richard D. Irwin Inc., 1972, $321 \mathrm{pp}$.

L'administrateur doit prendre des décisions face à des situations, à des problèmes souvent complexes par le nombre de paramètres qu'ils contiennent, de sorte qu'une solution intuitive comporte de grands risques.

La recherche opérationnelle, c'est l'application de l'analyse quantitative aux problèmes des décisions administratives. C'est la mathématique au service de la prise de décision. L'analyste ou le «spécialiste » de la recherche opérationnelle peut aider le dirigeant à systématiser l'analyse d'un problème et la prise de décision. Il vient épauler, à l'aide d'un outil de rationalisation, le dirigeant qui doit prendre une décision. C'est ce dernier cependant qui toujours prend la décision et non l'analyste qui intervient que pour mieux éclairer le dirigeant.

J'ose espérer que l'intention des auteurs n'est pas de remettre en doute la nature de cette relation lorsqu'ils écrivent qu'ils ne voient pas de distinction significative entre la science du management et la recherche opérationnelle.

Ecrit pour des non-mathématiciens qui désirent plus qu'une connaissance de surface ou des « recettes de l'analyse quantitative, cet ouvrage se veut être avant tout une discussion du sens commun et des possibilités d'utilisation des nombres en vue d'aider le dirigeant faisant face à des problèmes décisionnels.

Même s'il s'adresse à des «profanes », Operations Research for Managerial Decisions utilise quantité de formules mathématiques. Cependant, avant d'utiliser des concepts tels que le calcul différentiel et les probabilités, les auteurs prennent soin d'en présenter auparavant, les concepts de base.

Plus qu'une simple présentation théorique de la recherche opérationnelle, l'ouvrage à le souci du lecteur en ce sens qu'il cherche à développer, chez le lecteur, une certaine habileté dans la formulation des problèmes en termes de modèles mathématiques. Pour favoriser ce transfert, les auteurs mettent, tout au long du bouquin, l'emphase sur les techniques les plus utilisées dans leur application aux problèmes administratifs. Les exemples abondants et les problèmes qu'on retrouve à la fin de chacun des chapitres favorisent cet apprentissage.

$\mathrm{Au}$ plan du contenu, la programmation linéaire - terme utilisé pour décrire un grand nombre de techniques visant à optimiser ou trouver la meilleure valeur d'une fonction (objective function), en tenant compte des contraintes ou des exigences - retient l'attention principale des auteurs qui, auparavant, ont pris soin de nous instruire sur la nature de la recherche opérationnelle et d'esquisser les éléments principaux d'une théorie de la décision. Le dernier chapitre (chap. 9) du livre est consacré à la simulation comme moyen, de plus en plus intéressant à mesure que croît la complexité des systèmes, pour analyser les problèmes décisionnels.

En résumé, cet ouvrage convient à tout administrateur qui désire prendre un contact « concret» avec un outil d'analyse des problèmes impliquant une décision.

\section{Jean-Louis LANGEVIN}

\section{Université Laval}

Atlas de l'emploi : Ville et île de Montréal, par Claude Marois, Montréal, Les Presses de l'université du Québec, 1972, 184 pp.

Probablement seul un géographe est en mesure d'apprécier à sa juste valeur cet Atlas de l'emploi. Pour ma part, je trouve les 184 cartes intéressantes et non dénuées d'un certain esthétisme. II n'aurait certainement pas été inutile, cependant, que l'auteur explique davantage, ou mieux, la façon d'interpréter les ré- 